\title{
El proyecto de Folletería Mexicana del Siglo xIx: alcances y límites
}

Se explican el origen, desarrollo y resultados de la recopilación, en una base de datos, de 26500 títulos de folletos mexicanos del siglo XIX.

$\mathbf{P}$ ara un historiador dedicado al siglo XIX mexicano, el folleto no es un material desconocido. Casi todos los que se interesan en este periodo han consultado alguno de estos pequeños cuadernos impresos, de variados tamaños, sumariamente cosidos, forrados tan sólo con una hojita de color, a veces primorosamente ribeteada, que suelen tratar de los más diversos asuntos: desde un discurso ćvico hasta los méritos de una imagen milagrosa, desde una sentencia judicial hasta una tabla de tarifas aduanales, desde un modesto manual docente hasta un iracundo reclamo político, desde un presupuesto municipal hasta un texto de ley.

La folletería, como se le designa genéricamente, no es muy popular en las bibliotecas: suele ser la pesadilla de los empleados, que bregan para acomodarla en las estanterías debido a sus infinitas variaciones de tamaño. Y aun en los casos privilegiados en que se lo. gró obtener fondos para encuadernarla y reunirla en "misceláneas" - de conservación más segura y manejo más expedito-, su catalogación presenta dificultades inherentes no sólo a su formato, sino a su contenido y a las modalidades de su producción. 
En efecto, estas publicaciones, salidas de un sinnúmero de pequeñas imprentas o de talleres artesanales, nunca acataron las normas establecidas en el alto mundo editorial abocado a la producción de los libros. Sus pies de imprenta -a veces inexistentes- son frecuentemente incompletos, $y$ en ciertos casos, es preciso buscar en páginas interiores el dato que indica su fecha de publicación. Por otra parte, los personajes o los sucesos que aparecen en los folletos no alcanzaron siempre la relevancia necesaria para ser consignados en las obras de consulta general; por este motivo, ubicarlos resulta trabajoso y entorpece la labor de los bibliotecarios dedicados a su clasificación. Además, los asuntos tratados en los folletos son, en ocasiones, tan propios de la problemática de su momento que se disparan fuera de las categorias hoy en uso en las bibliotecas. Por todas estas razones operativas, la folletería no ha sido objeto de un marcado interés por parte de quienes son los encargados de acopiar y conservar los testigos escritos de nuestro pasado, al grado que, en numerosos casos, la folletería, siempre postergacla en su clasificación y catalogación, se amontona en los rincones más oscuros y olvidados de los acervos bibliográficos.

Este limitado interés por la folletería se ha visto reforzado por una opinión generalizada en el universo de la biblioteconomía y aceptada sin mayor reparo por los historiadores. Los folletos, asimilados a los pamphlets ingleses, serían un tipo de obra menor que en ciertos casos, pueden alcanzar un interés puntual, pero cuyo valor radica en ser el complemento de alguna publicación o documento de mayor relevancia.

Tal apreciación, sin duda pertinente en el contexto europeo en cl cual tomaron forma los primeros avances de la biblioteconomía moderna, no clebe trasladarse tal cual al caso mexicano, pues nada demuestra que las condiciones de desarrollo de la producción impresa hayan sido similares en ellondres que conoció el señor l)ewey y en el México decimonónico. Londres, como otras capitales europeas, cra, desde centenas de años antes, una plaza editorial importante en la cual se había desarrollado una floreciente producción de libros que descansaba en la existencia de un creciente público de lectores. La ciudad de México, aunque también tenía un conocimiento añejo de los trabajos de imprental, no podía considerarse, en el siglo XIX, como una plaza editorial de magnitud europea; no existía aquí un público lector comparable al citadino curopeo y, sobre todo, no se había desarrollado una cultura de la crítica política que brindara, al uso del pamphlet, la importancia social relevante que éste ocupó en la vida política de las monarquías europeas a finales del siglo XVIII y en los decenios siguientes.

La idea de estudiar con mayor cletenimiento la producción de folletos en México partió precisamente del reconocimiento de la diferencia de condiciones socioculturales entre México y Europa.

Por este motivo pareció pertinente tratar de explorar el mundo de los folletos con el fin de entender, en el contexto mexicano y no por asimilación 


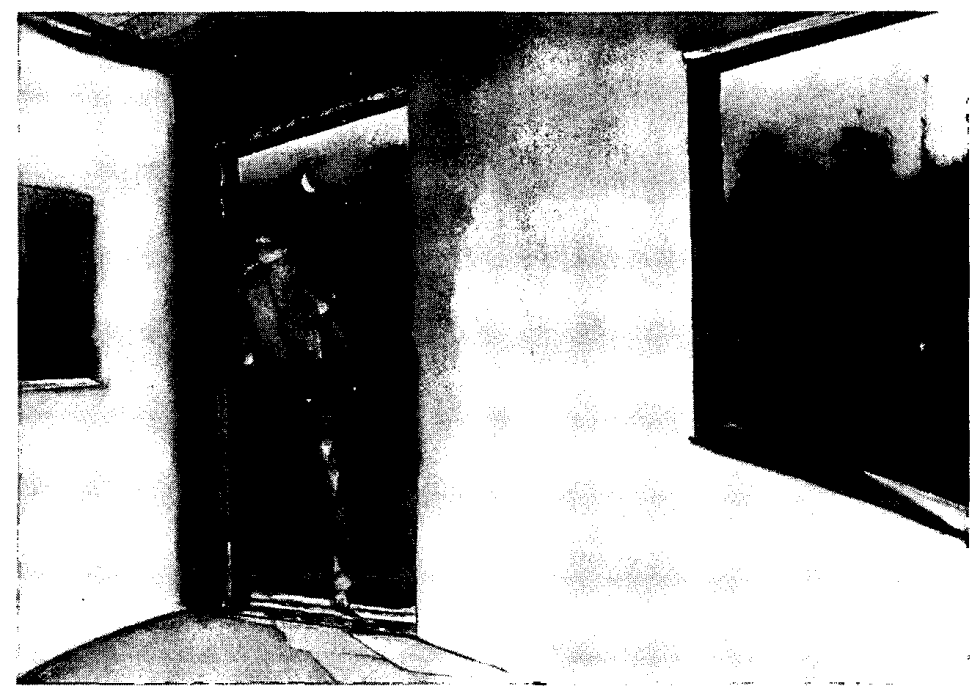

al caso inglés, lo que pudo significar aquí dicha forma de producción editorial.

Tal fue el propósito del proyecto de investigación que se ha venido desarrollando en el Instituto Mora desde 1994. En este marco se inscriben los trabajos aquí reunidos, que recojen las primeras conclusiones sobre este tema, producto de un programa de trabajo realizado durante poco más de dos años y medio, gracias al apoyo del Conacyt.

Como se indicó, la investigación fue iniciada en el Instituto Mora por los miembros del proyecto de Historia Política, quienes efectuaron algunos sondeos sobre las características de los folletos que conocían, los acervos en donde se sabía que existían coleccio- nes de ellos y los catálogos que sobre tales fondos se habían publicalo. En esta etapa preparatoria, se obturo la valiosa asesoría de la maestra María del Carmen Ruiz Castañeda, y se consultó a diversos conocedores de los grandes acervos bibliotecarios, en particular al maestro Miguel Ángel Castro, corresponsable del Seminario de Bibliografía Mexicana del Siglo XIX del lnstituto de Investigaciones Bibliográficas de la UNAM, quien nos invitó a incorporarnos a los trabajos en curso de realización en esta institución. También consultamos al maestro Luis Olivera, entonces encargado de la cattalogación faltante del fondo Lafragua de la Biblioteca Nacional; al doctor Manuel Ramos, director de la Biblioteca del Centro de Investigaciones Históricas 
de Condumex, y al licenciado Armando Rojas, abocado en aquel tiempo al ordenamiento del archivo histórico de Banamex.

Posteriormente, se procedió a la formulación de un proyecto de investigación específico que fue presentado ante el Conacyt, bajo la responsabilidad de Nicole Giron, para solicitar Ios apoyos necesarios a su realización.

Desde el inicio, la periodización contemplada fue ambiciosa, y abarcaba la totalidad del primer gran capítulo de la historia nacional: 1821-1910.

Para cubrir adecuadamente una extensión temporal tan amplia, se invitó a otros investigadores del Instituto ajenos al proyecto de Historia Política pero que, por sus intereses académicos peculiares, tenían cierta familiaridad con los materiales de estudio considerados.

Asimismo, se invitó a participar en el proyecto a investigadores que trabajaban en otras instituciones universitarias y habian manifestado su interés por las labores en vías de inicio. Así fue como se integró el grupo, constituido por diez investigadores del Instituto Mora: Graziella Altamirano, María Eugenia Arias, Nicole Giron, Laura Muñoz, Cecilia Noriega, Juan Ortiz, José Ortiz Monasterio, Laura Suárez de la Torre, Laura Solares, Guadalupe Villa, y cinco investigadores externos: el profesor Walter Brem, asistant curator de la Bancroft Collection en la Bancroft Library perteneciente a la Universidad de California, campus Berkeley; el doctor Brian Connaughton, de la Universidad Autónoma Metropolitana, Iztapalapa; la cloctora Virginia Guedea, del Instituto de Investigacio- nes Históricas de la UNAM; la doctora Antonia Pi-Suñer, de la división de Posgrado de la Facultad de Filosofía y Letras de la UNAM, y el maestro Javier Rodríguez Piña, de la Universidad Autónoma Metropolitana, Azcapozalco. A este grupo se unieron seis ayudantes de investigación, que fue posible reclutar con los fondos Conacyt. Ellos fueron los operadores efectivos de la captura o selección de datos, así como de su primera ubicación en categotías. Utilizaron para hacerlo el sistema de clasificación ideado por los investigadores y cuyas opciones se ofrecían a través de un programa de cómputo, diseñado para este efecto con la asesoría del ingeniero Adrián Medellín. Los ayudantes a quienes quiero expresar mi agradecimiento y transmitir las felicitaciones de todo el grupo de investigación fueron: Samantha Álvarez Macotela, Diana Corzo González, Alejandra Leal Martínez, Diana Macedo Alcalá, Lisbeth Medina Ortiz y José Miguel Villaseñor Bello.

La devaluación de diciembre de 1994 obligó a replantear buena parte del programa de trabajo previamente establecido y a sustituir con ingeniosidad y tiempo de captura suplementario una parte de los intercambios previstos con los acervos estadunidenses, sin embargo, después de 22 meses de un trabajo sistemático de selección y captura, se ha logrado constituir una base de datos que reúne aproximadamente 26500 registros, correspondientes cada uno al título de un folleto.

Tal cantidad de referencias -que por cierto no abarca la totalidad de los materiales existentes- indica que la 
producción de folletos en México durante el primer periodo de su historia nacional (1821-1910) fue algo más que una producción ocasional o secundaria. Fue una manifestación típica de la actividad editorial en este país y merece ser considerada como un conjunto significativo en sí.

No es posible, por el momento, cotejar esta producción particular contra la producción editorial general del pais durante el periodo considerado, pero se podrá proceder a comparaciones significativas cuando se termine el proyecto de Bibliografía General Mexicana del Siglo XIX que hemos mencionado. Lo que sí puede asegurarse, ya desde ahora, es que la folletería mexicana decimonónica constituye un corpus cuantitativamente considerable, y por ello digno de un examen detenido.

Los avances que presentan aquí apenas constituyen un primer levantamiento de algunas de las líneas de investigación que sugiere el corpus de la folletería mexicana decimonónica.

\section{¿CÓMO SE FUE INTEGRANDO LA BASE DE DATOS?}

Diversos factores explican que haya sido posible reunir, en un tiempo relativamente corto, una cantidad importante de datos.

Gracias a la generosidad y al espíritu de cooperación de algunas instituciones, fue posible acceder a los inventarios computarizados que poseen diversos depósitos nacionales o extranjeros. Inicialmente fue la biblioteca Condumex que, como se sabe, con-

El proyecto de Folletería MeXicana serva grandes cantidades de publicaciones decimonónicas. Posteriormente la Biblioteca Nacional del Instituto de Antropología e Historia (Biblioteca Eusebio Dávalos), en donde se lleva a cabo un importante proceso de automatización. Y, finalmente, la Bancroft Library, cuya cooperación ha sido determinante en el avance de nuestro proyecto. Quiero agradecer aquí, con todo énfasis, el apoyo generosamente prestado por los directores y conservadores de estos importantes acervos cuyas colecciones constituyen la parte medular de lo incluido en nuestra base de datos.

También se obtuvo, indirectamente, una aportación computarizada de la Biblioteca de la Universidad de Texas en Austin a través del NISC-DISC editado por esta institución como instrumento de consulta de los títulos reunidos en la Nettie Lee Benson Collection. Su utilización nos fue facilitada por la Biblioteca de El Colegio de México.

En todos estos casos nuestras labores de "captura" se vieron considerablemente aligeradas. En efecto, el trabajo ya no consistió en capturar los datos y el texto de los títulos, sino en eliminar de la información transferida los registros que no correspondían ni a la época ni al tipo de obras que queríamos reunir.

Para llevar a cabo esta considerable tarea de selección fue preciso un tratamiento informático de los diferentes "paquetes" de datos recibidos, no siempre codificados en el mismo lenguaje ni tratados con el mismo software.

Pero, además, fue preciso distinguir entre tanto material los títulos que sí correspondían a folletos. 
Para ello era necesario saber con precisión qué era o no un folleto. La discusión de esta definición fue ardua. Nos permitió conocer las fluctuaciones considerables que las propias normas de biblioteconomía atribuyen al tamaño de los folletos, con variaciones del $100 \%$, puesto que para unos son folletos las publicaciones impresas, no periódicas, que alcanzan hasta 49 páginas $y$, para otros, se consideran como folletos las publicaciones impresas, no periódicas, que alcanzan hasta 100 páginas.

Finalmente, y después de haber revisado ejemplares publicados en diferentes momentos del siglo XIX, nos adherimos a la segunda definición, y aceptamos como folleto, independientemente de su temática, cualquier publicación no periódica, impresa en México, que fuese superior a dos páginas e inferior o igual a 100 . Asimismo, se aceptó cualquier publicación publicada en el extranjero con las mismas características, sobre un tema mexicano, durante el mismo periodo.

También se acordó, con base en el examen de casos fehacientes, incluir publicaciones que podian alcanzar un máximo de 140 páginas, en particular del periodo de 1821 a $1840-50$, durante el cual prevaleció la costumbre de añadir a los textos publicados como folleto documentos probatorios anexos, ${ }^{1}$

1 El caso es particularmente frecuente entre los folletos jurídicos, que alcanzan un número considerable de páginas. A este respecto véase en este mismo número el artículo de Jaime del Arenal, quien asegura que, en las colecciones de la Escuela Libre de Derecho, figuran folletos de hasta 250 páginas. Motivo por el cual, en traba- sin que el consecuente abultamiento del número de hojas de aquellos libritos modificase para nada la percepción de éstos como "folletos" u "opúsculos", como se les nombraba también.

La dificultad para establecer un criterio preciso, relativo al número de páginas, procede de la naturaleza peculiar del "folleto", cuya extensión varía en función del espacio necesario a su autor para exponer la cuestión que le preocupa. De modo que encontramos folletos de menos de diez páginas, como por ejemplo la Defensa que presenta Pedro MacCormick ante el tribunal de la opinión pública, rechazando las injurias que le infiere el señor don Mariano Pérez Bolde, en la hoja denominada "A la sensata sociedad", publicado en Querétaro por la Tipografia de González y Compañía, en 1893 y que tiene siete páginas; o la Novena carta pastoral del ilustrisimo obispo de Michoacán, licenciado don Clemente de Jesús Munguía, exponiendo a los fieles de su diócesis las razones que tuvo para protestarcontra el decreto de 25 de junio de este año, sobre expropiación de fincas pertenecientes a corporaciones eclesiásticas, publicada en Morelia en 1856 y que ocupa ocho páginas.

Pero también encontramos el $R e$ glamento de debates del Ayuntamiento de Puebla de Zaragoza, de 32 páginas, que fue publicado en Puebla por la Imprenta de Corona Cervantes en el

jos ulteriores se tendrá que relativizar aún más la importancia de los criterios formales usados en la biblioteconomia y privilegiar los conceptos ligados a la temática de los materiales consiclerados. 
año de 1892; o el Proyecto para la formación de la Compañía Nacional de Hierro y Acero, publicado en la ciudad de México por la Tipografia de Bouligny y Cía. en 1892, que alcanzó 34 páginas.

Asimismo, encontramos el Quinto calendario impolítico y justiciero para el año de 1857, arreglado al meridiano de México, de 70 páginas, publicado por la imprenta de Juan R. Navarro en la ciudad de México; o los Apuntes para las lecciones de jurisprudencia médica, explicadas en la cátedra respectiva de la escuela especial de jurisprudencia de la Sociedad Católica, extractadas de algunos autores de medicina legal (Zachias, $H$., y Carpio, Mata, Briandy Chaude, Rosell y Sedillot) por el licenciado $M$. Mancilla, publicado en Guadalajara en 1879 , que tenía 68 páginas y, por fin, uno de tantos alegatos o querellas e informes jurídicos publicado en folleto, el Informe en estrados, producido ante el Supremo Tribunal de Justicia del Estado, por el licenciado Isidro Rojas, apoderado jurídico de la Compañía Restauradora del Mineral de Veta Grande en el juicio sumario que sobre procedencia del denuncio de la mina La Flor ha seguido contra aquella negociación el licenciado don Benito Garza, diciéndose apoderado de la Cía. Minera "La Asturiana". Se publica además otro documento relativo al mismo negocio, salido de la imprenta de La Rosa, en la ciudad de Zacatecas en 1892. Existen de él dos ediciones diferentes: una de 94 páginas y otra, con el mismo título, impresa bajo la misma razón social, pero que alcanza las 124 páginas, sin que podamos saber-puesto que no manejamos directamente los ejemplares-si se trata de dos folletos de formato diferente, siendo más pequeño uno que otro, lo cual explicaría el aumento del número de páginas, o si el más largo tiene el mismo formato que el otro, pero incluye en su interior algún documento probatorio ausente en el primero.

Podemos considerar este último ejemplo como excepcional, y afirmar que el $90 \%$ de los títulos incorporados a nuestra base corresponden a obras de menos de 70 páginas.

Acabo de indicar que no trabajamos directamente con los ejemplares de los folletos. Es necesario aclarar este punto para no inducir a confusión a los posibles utilizadores de la base.

La base de datos de Folletería Política Mexicana del Siglo XIX que se quería constituir en el Instituto Mora nunca pretendió ser un instrumento de biblioteconomía exhaustivo, sólo respondió al deseo de facilitar al historiador la localización de materiales útiles para sus investigaciones. De modo que el reto fue concentrar una información que estaba sumamente dispersa y generar categorías de clasificación que se desprendiesen de los materiales recopilados sin tener que recurrir a conceptos predeterminados que son, muchas veces, o demasiado generales, o improcedentes para los materiales históricos.

El propósito de la base fue, por tanto, acumular la mayor cantidad posible de información, y ubicar con precisión el origen de la misma.

Si un título aparece en el inventario computarizado de la biblioteca Con- 
dumex o de la Bancroft Library, damos por sentado que el ejemplar mencionado existe efectivamente en este acervo, y que el investigador podrá localizarlo en dicho punto. No nos competen las labores propias de los bibliotecarios, que deben ubicar los ejemplares de sus colecciones, conocer sus características físicas, proceder a su catalogación, cuidar de su conservación.

Por este motivo, cada uno de nuestros registros incluye la indicación del fondo en donde se localizó el título mencionado, considerando como confiable dicha información.

El mismo procedimiento se aplicó para los acervos cuyo rastreo se hizo a través de catálogos publicados o mecanografiados, como es el caso de la Biblioteca Pública de Guadalajara; del Fondo Basave de la Biblioteca México; de la Sutro Collection de San Francisco; de la colección de documentos mexicanos de la Universidad de Yale; del catálogo del fondo Lafragua de la Biblioteca Nacional, establecido por Lucina Moreno; del de folletos del archivo Porfirio Díaz conservado en la Universidad Ibero-Americana, o del fondo reservado de la Biblioteca Orozco y Berra del INAH.

Para evitar equívocos, es preciso recalcar que sólo utilizamos catálogos ya publicados o inventarios computarizados. No disponíamos ni de un equipo suficientemente numeroso, ni del tiempo necesario para ir a levantar, a mano, y de una en una, las fichas bibliográficas pertinentes que se hallan esparcidas entre muchos otros materiales en los tarjeteros de los acervos.
Al iniciar la recolección de datos para la base, se nos planteó la dificultad de querer discernir a priori lo que era folletería política. En vista de la imposibilidad de hacerlo, ya que el folleto responde a una infinidad de intenciones y se inserta en una coyuntura que siempre tiene algún matiz político, aunque hoy en día no lo distingamos claramente, decidimos recoger todos los folletos posibles para entresacar más adelante los que fueran políticos. De este modo cambió ligeramente el enfoque de nuestro trabajo, y puede decirse que nos abocamos a conformar una base de datos de Folletería Mexicana del Siglo XIX, reservando para trabajos ulteriores la apreciación del significado político de las variaciones registradas en la producción de folletos consagrados a un determinado tema.

En cuanto a las categorías y rubros, utilizados como estructura conceptual de la base, unas y otros permiten un acceso ordenado a sus materiales. Fueron generados a partir del vocabulario utilizado en los folletos y procurando la mayor proximidad con nuestra terminología actual.

Estas divisiones clasificatorias facilitan la recuperación de los materiales al fragmentar la base en pequeñas unidades específicas, y permiten apreciar, a lo largo del tiempo, las variaciones en la producción de folletos sobre determinados temas.

La base se subdivide en doce categorías que, a su vez, agrupan un total de 154 rubros. Constituyen un listado relativamente compacto que, dispuesto en columnas, cabe en una sola hoja recto-verso, y nos permitió acomodar los 28000 registros actuales. 
Para no volver inmanejable esta masa de información, hemos decidido aplicar sólo tres posibilidades de clasificación por folleto. Este procedimiento nos permitió ubicar un mismo folleto, cuyo título podia interesar por diferentes motivos, en diversas subdivisiones o rubros. Tomemos un ejemplo, el folleto titulado: Estatuto de la Asociación Americana de Salubridad Pública y extracto del reglamento de la comisión ejecutiva, publicado en la ciudad de México, impreso en la antigua imprenta de Murguía, en 1892 , que tiene 16 páginas.

Fue clasificado en el rubro: Estatutos-Reglamentos interiores de la categoría Obra jurídica. También fue adscrito al rubro: Asociaciones, de la categoría Obra de tema social. E igualmente fue incorporado al rubro: Medicina, de la categoría Obra científica. De tal manera que puede ser recupe: rado tanto por el investigador interesado en los aspectos legislativos como por el que se ocupa de historia de la ciencia o el que observa el desarrollo de las asociaciones, $\tan$ característico de la vida decimonónica, suponiendo que cada uno de estos investigadores se limitara a consultar la base sólo en los rubros de su interés directo. Pero es obvio que, al cruzar la información ubicada en diferentes rubros, el historiador descubrirá pistas de investigación y podrá derivar de ellas nuevas consultas apropiadas para el caso que lo moviliza.

También es necesario apuntar que el investigador deseoso de consultar la base de datos, sacará gran provecho de las indicaciones relativas a su diseño y funcionamiento proporcionadas por el Manual del usuario que será puesto a su disposición, aunque nos hemos esforzado por diseñar, aun sin este aditamento indispensable, un programa de consulta amigable que no presente mayores dificultades de utilización.

En este momento, el diseño de la base no está completamente acabado; todavía falta perfeccionar las modalidades de recuperación hoy previstas, que son las siguientes:

1. Por año.

2. Por nombre de autor o pseudónimo cuando lo hay.

3. Por título.

4. Por lugar de edición.

5. Por editor.

6. Por número de páginas.

7. Por región a la cual se refiere el folleto.

8. Por categoría.

9. Por rubro.

10. Por fondo bibliográfico.

Con la posibilidad de combinar estos diversos factores entre sí.

\section{LÍMITES}

Si lo expuesto arriba nos permite apreciar los alcances de la base de datos de Folletería Mexicana del Siglo xIx, también es necesario tener muy claras sus limitaciones para no extraer de ella conclusiones imprudentes.

En primer lugar, es preciso recalcar que el corpus reunido hasta hoy está incompleto: existen materiales que no han sido incorporados a la base de datos, entre otros motivos porque el 
equipo de cómputo del que disponíamos no podía soportar un crecimiento indefinido de información y -además-no era nuestra intención efectuar una recopilación exhaustiva de la folletería decimonónica, sino explotar el universo que la constituía.

La investigación demostró que tan sólo en los acervos nacionales existe una gran cantidad de información, aún no totalmente catalogada, que podría recuperarse si se generaran, con los bibliotecarios encargados de su conservación, los programas de cooperación adecuados.

No perdamos de vista el hecho de que nuestra base refleja, antes que la producción de los folletos en el pasado siglo, su presencia en los acervos de las bibliotecas, es decir, su conservación. Precisamente por este motivo nuestras fuentes han sido: o bien catálogos publicados o bien inventarios computarizados del contenido de los acervos bibliotecarios.

Cuando vayamos a inferir de la información que arroja la base observaciones sobre la importancia de tal o cual tipo de producción, deberemos tomar en cuenta siempre esta doble distorsión, reflejo de un gran número de avatares.

¿Hubiera existido el fondo Lafragua sin el empeño coleccionista y la sensibilidad política de un José María Lafragua y sin la circunstancia providencial de que entre sus cargos políticos le tocara dirigir en algún momento la Biblioteca Nacional? ¿Hubieran existido las colecciones de la Bancroft Library, sin el afán totalizador y la considerable fortuna personal, del historiador-empresario que las constituyó?
Podríamos observar lo mismo acerca de las colecciones de la Universidad de Texas o de la Sutro Collection. Sin duda la capacidad de compra del dólar estadunidense tiene mucho que ver con el hecho de que un gran número de obras mexicanas hayan ido a parar a acervos situados del otro lado del río Bravo.

Por otra parte, las grandes bibliotecas europeas albergan importantes colecciones de publicaciones referentes a México, ya que nuestro país fue, por diversos motivos, objeto del interés público -o particular-en aquellas naciones. Además, algunas ciudades europeas llegaron a ser, en el siglo XIX, verdaderas plazas editoriales en idioma español que generaban publicaciones exportadas para su venta hacia Latinoamérica.

Sería deseable, puesto que la presente investigación demostró la relevancia de la folletería en México y estableció que este género editorial puede constituir un objeto de estudio autónomo, preparar una segunda fase de investigación destinada a recabar el universo completo de los folletos y analizar a profundidad el sentido y las implicaciones de esta producción.

Otra consideración es importante para señalar las limitaciones de nuestro proyecto: al trabajar solamente a partir de los títulos de los folletos, es posible que hayamos distorsionado la información que éstos contienen.

Sucede que algunos títulos, de vena satírica, son poco explícitos y hasta dificiles de entender si no se tiene acceso al texto completo. Algunos otros son confusos o misteriosos, y los contenidos que amparan pueden ha- 
ber ido a parar a algún rubro sin relación con su significado real.

Tales peligros son el costo, inevitable, de un trabajo efectuado sin consultar efectivamente los documentos; pero, ¿podía pensarse en ello frente a una dispersión geográfica tan considerable de los fondos de consulta y tratándose de una cantidad importante de publicaciones, mismas que debían ser identificadas o capturadas en un breve lapso de tiempo?

A pesar de este serio escollo, creemos que $95 \%$ de los materiales incluidos en la base han recibido una ubicación aceptable.

También es posible, sobre todo en la categoría de las obras literarias o de las cientificas, que hayamos incorporado como "folletos", impresos que en realidad son libros; libros de pocas páginas, es cierto, pero editados en un papel de gran calidad, con una tipografía esmerada, una encuadernación de tapa dura e ilustraciones elegantes en forma de grabados o de fotografias. Sin embargo, acerca de este último punto es necesario precisar que auténticos folletos, es decir, publicaciones baratas, también incluyen a veces tablas, fotografias o grabados.

Aun con estos inconvenientes, que trataremos de compensar a la hora de la depuración final, creemos que la base de datos de Folletería Mexicana del Siglo XIX constituye un valioso instrumento de investigación para rastrear información sobre temas específicos. Asimismo, abre un acceso sistematizado a un campo editorial hasta ahora subvalorado. Pues si bien es cierto que nunca podremos conocer exactamente la producción edito- rial de los folletos, ya que faltan los datos contables o de archivo que nos permitirían hacerlo, también es cierto que, a través de una base de datos como la que hemos constituido, es posible obtener estimaciones significativas.

El número de registros recalados hasta ahora muestra que los folletos ocuparon un espacio editorial no despreciable, intermedio entre el de los libros -el camino real de las letras impresas- y el de las publicaciones periódicas. Queda por dilucidarsu función social, sobre todo si consideramos el ámbito de la discusión política, pero la importancia misma de su producción -un promedio anual de 233 folletos al final del siglo y de $44\{7$ al principio de la época independienteindica que no se trató por ningún concepto de un género segundario. Por el contrario, queda claro que los folletos respondieron a las necesidades específicas de comunicación de una sociedad que no se limitó a leer y producir libros, ni a leer y producir periódicos.

Los estudios que de un modo pionero emprendió Jaime del Arenal sobre el folleto juridico; ${ }^{2}$ las conclusiones que Brian Connaughton ha sacado del estudio de los sermones religiosos publicados como folletos; las perspectivas que Antonia Pi-Suñer ha trazado a partir del análisis de los opúsculos relacionados con la "cuestión española", son ejemplos que ilustran algunas de las posibilidades abiertas por el estudio sistemático de la folletería mexicana decimonónica.

2 Arenal, "Hacia", 1987. 


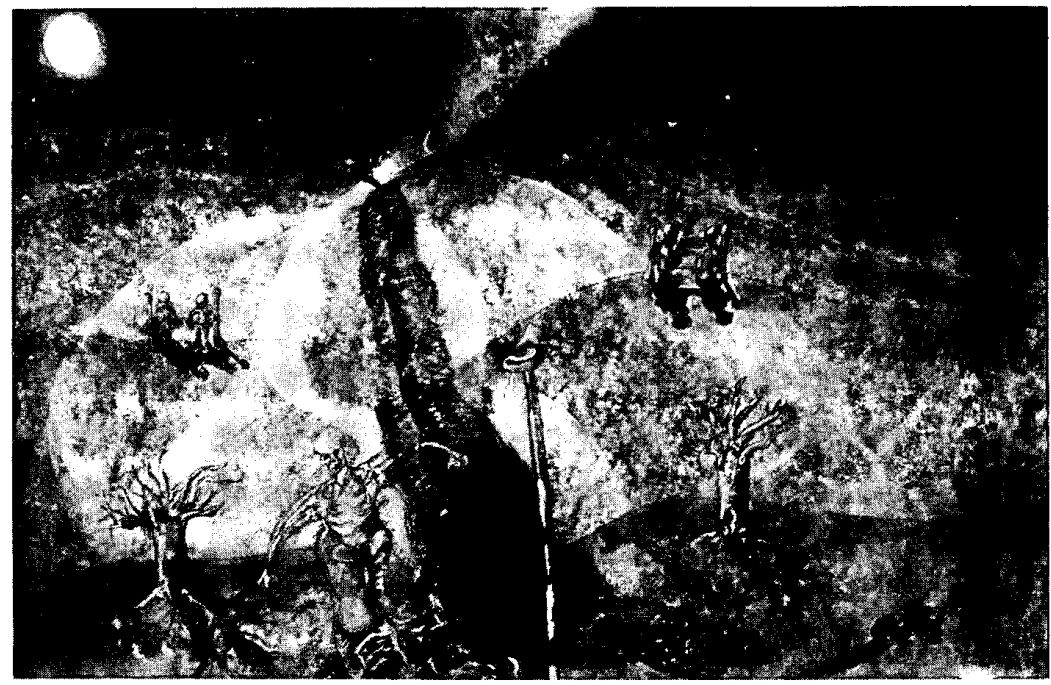

La impresión de los folletos era generalmente costeada por sus autores, mismos que acordaban con el impresor el volumen de la edición según la difusión que pensaban dar a su obra.

Es extremadamente dificil conocer el tiraje ${ }^{3}$ de tales ediciones, puesto que los folletos, al igual que los libros del siglo pasado, jamás incluyeron datos a este respecto. Sin embargo, una investigación más profunda sobre el mundo editorial que aflora a través de los pies de imprenta, permitiría

${ }^{3}$ Hemos encontrado un folleto publicado en 1891, cuyo título brinda esta indicación excepcional: Serie cronológica de los deanes de la catedral de México. Apuntes porJesús Galindo y Villa, Imprenta de la Voz de México, México, 77 pp. Nota: reimpresión de 50 ejemplares. relacionar los editores e impresores - cuya dirección está frecuentemente mencionada en la portada de los folletos- con los levantamientos censales de artesanos, o los padrones de causantes conservados en los archivos municipales, arrojándose de este modo alguna luz sobre sus actividades.

El hecho de que los grandes editores no hayan despreciado la publicación de folletos - pues son numerosos los que salieron de los talleres de Ignacio Cumplido o de Vicente García Torres por ejemplo- nos hace pensar que producirlos no debió ser mal negocio.

En cuanto a la difusión de los folletos, debió ser importante, ya que encontramos, en la década de los cuarenta del siglo XIX, por lo menos dos 
decretos que contienen disposiciones tarifarias específicas y más económicas para este tipo de impresos. ${ }^{4}$ ¿Será porque viajaban bajo este formato las leyes o disposiciones reglamentarias emitidas por diversas oficinas gubernamentales?

En verdad sabemos muy poco a este respecto, y convendría estudiar con mayor detenimiento las cuestiones materiales ligadas a la producción, la difusión y el impacto de los folletos en la vida decimonónica.

Ésta es otra de las investigaciones a que nos invita la base de datos de Folletería Mexicana del Siglo XIX. Estudio que permitirá utilizar información referente no sólo a la capital de la república, sino a las ciudades del interior para las cuales también disponemos de la localización precisa de un gran número de talleres de imprenta.

4 El decreto de 24 de octubre de 1842 sobre tarifas para cobrar el porte de la correspondencia, contemplaba cuatro tarifas diferentes. En la segunda aplicable a los envios de 16 a 100 leguas se especifica: "los periódicos, sea cual fuere la distancia, pagarán 4 reales por cada 100 pliegos, y los folletos o impresos sueltos la 8a. parte de lo que designa esta tarifa, fijándose en $1 / 2$ real el minimum de cada pieza". Dublán y Lozano, Legislación, 1876-1912, t. 4, p. 297.

El decreto de 11 de octubre de 1848 derogaba esta última disposición y establecía:

Art. 1. Los periódicos o impresos ahora conocidos con el nombre de folletos pagarán de porte a razón de $1 / 2$ real por libra de peso que contengan.

Art. 2. Los que no lleguen al peso de 4 onzas, pagarán sin embargo, $1 / 8$ de real y no se cobrará cosa alguna por las intermedias de exceso que llegare a haber entre cada 4 onzas. Dublán y Lozano, Legislación, 1876-1912, t. 5, p. 486.
UNA OJEADA A LOS FLUJOS DE LA FOLLETERÍA DECIMONÓNICA

El examen de los flujos de esta producción editorial, de la cual sólo daré pocos ejemplos para no alargar excesivamente este artículo, considera dos variables, el volumen de la producción registrada y su distribución en el tiempo.

Desde los inicios del diseño de la base, el periodo global de estudio; 18211910, fue dividido en seis subperiodos de una duración más o menos equiparable. Procuramos dara los cortes temporales cierta congruencia con la periodización política: el primero va de 1821 a 1836; el segundo de 1837 a 1851; el tercero de 1852 a 1867 ; el cuarto de 1868 a 1880; el quinto de 1881 a 1892, y el sexto y último de 1893 a 1910.

Esta división temporal permitió analizar provechosamente la producción de folletos para cada una de las categorías de la base, poniendo en evidencia el crecimiento o el declive de las distintas temáticas a lo largo del siglo y su importancia relativa para cada subperiodo.

Así se hicieron patentes ciertos desfases entre los picos de producción de un determinado tipo de folletos y las fechas que la historiografia ha consagrado como significativas para los asuntos que tratan.

Si tomamos un ejemplo en la categoría de las Obras eclesiásticas -que será estudiada más detalladamente por Laura Suárez de la Torre y Javier Rodríguez Piña-, podremos observar que el pico de producción de folletos relativos a los bienes de la Iglesia, se sitúa en 
el periodo $1837-1851 \mathrm{y}$ no en el periodo $1852-1867$ como podría pensarse, puesto que este último incluye los. años decisivos de 1856 (con la ley de desamortización de los bienes de las comunidades civiles y religiosas, en junio) y de 1859 (con la ley de nacionalización de los bienes de la Iglesia, en julio). Dicho rubro arroja las siguientes cifras: entre 1821 y 1836, 27 folletos; entre 1837 y 1851, 129 (un brinco enorme: $477 \%$ en relación con el periodo anterior); entre 1852 y 1867 , 78 ; entre 1868 y 1880 , doce; entre 1881 y 1892 , siete, y entre 1893 y 1910 sólo uno. Lo cual parece indicar que la cuestión de los bienes de la Iglesia, de la legitimidad de su posesión por el clero, del uso que convenía hacer de ellos, fue un tema ampliamente debatido mucho antes de que se generaran las leyes que zanjaron dichos problemas.

Observamos una evolución paralela en el rubro llamado Documentos políticos, Iglesia-Estado, que agrupa Ios siguientes registros: entre 1821 y 1836,163 folletos; entre 1837 y 1851 , 239 (o sea $146 \%$ más que en el periodo anterior); entre 1852 y 1867,125 ; entre 1868 y 1880 , catorce; entre 1881 y 1892 , diez, y entre 1893 y 1910 caemos a cuatro.

Aquí la evolución de los flujos indica también que el asunto de las relaciones Iglesia-Estado conoció, por lo menos entre los autores del folleto, su momento de mayor discusión antes de 1851 y no, como lo marca la periodización usual, entre la revolución de Ayutla y el imperio de Maximiliano. ¿Permite esta constatación avanzar la hipótesis de que los autores de folle- tos constituyeron un grupo que se habría anticipado a sus contemporáneos en la detección de las problemáticas políticas de su tiempo? Ciertamente no, la variedad de cuestiones abordadas en la folletería demuestra que participó de su producción el más amplio surtido de personajes, pero podemos interrogarnos sobre nuestra percepción del tiempo de maduración social de un problema, en este caso el importantísimo de las relaciones Iglesia-Estado.

Tomemos otro ejemplo en la categoría de las Obras económicas, en la cual, el rubro más nutrido es el de Contribuciones-Impuestos. Ahí encontramos que: entre 1821 y 1836 , se produjeron 254 folletos; entre 1837 y 1851 , 203; entre 1852 y 1867,94 ; entre 1868 y 1880,115 ; entre 1881 y 1892 , 81 , y entre 1893 y 1910,110 . Con lo cual podemos afirmar que la cuestión fiscal fue el tema económico que generó mayor preocupación entre los mexicanos a lo largo del siglo XIX. Ni siquiera el rubro de Comunicaciones, que en nuestra categorización agrupa los folletos relativos a correos, ferrocarril, puertos, telégrafos y otros, capitalizó un número similar de títulos. Veámoslo: entre 1821 y 1836 encontramos en Comunicaciones, 31 folletos; entre 1837 y 1851,74 ; entre 1852 y 1867,62 ; entre 1868 y 1880,159 (un aumento de $250 \%$ en relación al periodo anterior); entre 1881 y 1892,133 , y entre 1893 y 1910, 109.

En este rubro el gran salto cuantitativo ( $250 \%$ de aumento) se produce en el periodo de la república restaurada. Constatamos un ligero declive durante el porfiriato, considerado tradi- 
cionalmente como el momento de mayor avance en las comunicaciones de todo tipo.

Ello no puede dar pie a reconsiderar la importancia del desarrollo de las comunicaciones en el porfiriato, pero sí nos invita a revalorar el dinamismo -aunque sea en proyectos- del último periodo presidencial de Juárez y de la presidencia de Lerdo de Tejada.

Por contraste, si nos detenemos en el rubro llamado Empresas-Sociedades mercantiles, observamos que presenta hasta 1910, una tendencia constantemente ascendente: entre 1821 y 1836 encontramos, 17 folletos; entre 1837 y 1851,22 ; entre 1852 y 1867 , 76 , un aumento de $245 \%$ (más notable aún porque se produce durante un periodo de guerra, civil y extranjera); entre 1868 y 1880,69 folletos; entre 1881 y 1892,120 , y entre 1893 y 1910 , 134.

Es posible que el ligero descenso correspondiente al periodo 1868-1880 -siete folletos menos que en el subperiodo anterior- se deba a un desequilibrio estructural de la base que registra, en este tramo, el menor número de títulos: 2869 contra 3786 para el inmediatamente anterior, y 3327 para el inmediatamente posterior.

Hemos mencionado arriba las posibles limitaciones de nuestro trabajo: se puede legítimamente formular interrogantes sobre la confiabilidad actual de la base de datos.

El juzgarla "completa" o "incompleta" implica cierto subjetivismo, y existen elementos razonados que permiten cuestionar la información numérica que de ella se desprende.

Por ejemplo, el no haber manejado información relativa al contenido de algunos grandes acervos, como la segunda parte de la colección Lafragua de la Biblioteca Nacional de México, correspondiente a los años 1854-1876, que todavía no está disponible en inventario computarizado ni en catálogo impreso, o bien el haber ignorado las colecciones de la Escuela Libre de Derecho, todavía en proceso de automatización al día de hoy y que, por tanto, no pudieron ser recopiladas en nuestra base.

El panorama de lo no incluido en la base se amplía todavía más si consideramos los contenidos no catalogados de numerosos acervos bibliotecarios en el interior de la república, o bien la información que, catalogada en tarjeta, no ha sido objeto de una publicación especial, o para la cual apenas se inicia un proceso de automatización y tampoco pudo ser considerada por nosotros.

Aun así, la lectura de los flujos que la base de datos señala muestra una posible utilización de este instrumento no sólo como un aparato de consulta bibliográfica que permite localizar al mismo tiempo los títulos que interesan al investigador y el fondo en clonde consultarlos, sino que puede generar una información nueva sobre la situación de aquellos impresos dentro de las tendencias de la producción editorial de su momento o en el largo plazo.

Si tomamos en cuenta que el costo de la impresión de los folletos corría totalmente a cargo del autor-ocle la institución que lo mandaba imprimir-, está claro que los flujos de producción observados no resultan de las tendencias especulativas de un posible mer- 
cado editorial, y son sólo el reflejo de las preocupaciones o del parecer de sus autores, inseparables -suponemos-de las del público que se tomaba el tiempo de leerlos.

A través de la folletería, podemos entrar en contacto con una parte de los debates que agitaron a la sociedad mexicana. Quizá no se trate de las grandes cuestiones especulativas de la filosofía política o de los reclamos de principio que se ventilaban en el aristocrático universo de los libros. Tampoco se trata de las diarias peroratas que ocupaban las columnas de los periódicos, muchas veces abiertas a las traducciones extranjeras. Se trata casi siempre de inquietudes vernáculas, de preocupaciones concretas, estrechamente ligadas a acontecimientos inmediatos. Tanto es así, que fue preciso abrir en nuestra base una categoría titulada: Obras referidas a periódico. Misma que agrupa un total de 554 títulos, una cantidad insignificante frente a los 26500 registros de la base, pero cuyo flujo en el rubro Folletos relacionados con un artículo periodístico, señala una línea tenue pero constante: entre 1821 y 1836,33 folletos; entre 1837 y 1851,93 (un fuerte salto, $221 \%$ ); entre 1852 y 1867,73 ; entre 1868 y 1880,41 ; entre 1881 y 1892,50 , y entre 1893 y $1910,36$.

Para terminar, nos falta formular una pregunta $¿$ Hubo una edad de oro de la folletería en México? Muchos piensan que la mejor época de este tipo de producción corresponde a la década de los cuarenta o de los cincuenta del siglo XIX. Sin embargo, los datos arrojados por nuestra base dan la preeminencia al primer subperiodo, el de
1821 a 1836, que demuestra claramente el derroche de actividad editorial ${ }^{5}$ que acompañó a la independencia. Los folletos se beneficiaron de la explosión de publicaciones que propició la libertad de imprenta entonces promulgada, aunque muy pronto restringida. ${ }^{6}$ En números absolutos, el año de 1822 registra la mayor producción del siglo: 1026 títulos, cifra muy superior al promedio del subperiodo 1821-1836, que es de 447 títulos por año, el más elevado en la base.

Aun teniendo presentes las limitaciones de nuestro trabajo no dejan de ser significativos estos números.

A lo largo de los noventa años que hemos estudiado (1821-1910) se observa una disminución en la producción de los folletos que, expresada en promedio anual de producción, pasa, para los seis periodos delimitados, de 447 folletos a $290,236,220,277$ y 235 , respectivamente.

Para sorpresa de muchos, la producción de folletos no desapareció a fines del siglo, aunque para entonces había aumentado considerablemente la publicación de libros y la de revistas periódicas, científicas o literarias, sin contar que se había consolidado cl emporio de la prensa diaria, sólidamente afianzado en la opinión pública.

Lo que indica nuestra base para el último subperiodo estudiado (18931910) es un cambio de distribución en la temática de los folletos, un cambio

\footnotetext{
"Bachman, "Panfletos", 1971, pp. 552-538.

"Un ejemplo de ello se puede hallar cn la producción de folletos de José Joaquín Fernández de Lizardi, el "Pensador mexicano", recopilados en el vol. XIII de sus Obras, 1995.
} 
cualitativo en sus contenidos. Menos actas o representaciones parlamentarias (se pasa de 262 a principios del siglo a 38 al final); menos polémicas políticas (1 485 contra 81); menos opúsculos sobre política exterior $(571$ contra 46); a la inversa, se registra un número creciente de cartas pastorales (de 60 a principios de siglo, a 103 al final), o de expresiones del culto mariano (de 70 a 122). Se manifiesta una progresión en la publicación de manuales y tratados científicos (32 a principios de siglo contra 269 al final), o de publicaciones realizadas por asociaciones científicas (se pasa de siete a $155)$, así como de folletos relativos a cuestiones de tierras y aguas ( 23 contra 114). También los alegatos, querellas, informes de estrado, agrupados en un solo rubro, dan un brinco espectacular (pasando de 97 a principios de siglo a 409 al final), acompañados por los estatutos y reglamentos interiores, que pasan de 43 a 217 , en contraste con la evolución registrada en el rubro de Leyes y decretos, que baja de 484 al principio del siglo a 142 apenas al final, probablemente porque los textos de ley que se promulgaron entonces circulaban por conducto de los diarios oficiales estatales o en el de la federación.

Todas estas indicaciones numéricas nos invitan a tomar en cuenta la evolución en el largo plazo, tan esencial para detectar los cambios culturales. Nos alertan sobre aparentes desfases o contradicciones, guiándonos hacia explicaciones más finas de lo ocurrido; por ejemplo, hacia la percepción de las nuevas formas de comunicación y de organización adopta- das en la administración pública o en el mundo de los negocios, ya que el decaimiento observado en el rubro Proyectos económicos (34 folletos a principios de siglo contra 17 al final) se encuentra en completa contradicción con el desarrollo del país, y debe traducir, más que una disminución de las propuestas económicas, una canalización diferente de este tipo de documentos.

\section{CONCLUSIÓN}

Mucho camino queda todavía por andar en el proyecto de Folletería Política Mexicana del Siglo XIX. La reflexión sobre la naturaleza de lo político que habíamos esbozado como uno de los objetivos del proyecto, seguramente se quedará en el tintero, pues nos rebasó la enormidad de la documentación, tan distante de nuestras estimaciones iniciales.

Tampoco creíamos, al emprender este proyecto, que sus resultados nos Ilevarian a planear una segunda etapa de investigación que incluyera la formulación de un programa de cooperación con los bibliotecarios de la república.

Sin embargo, aun con todas las limitaciones que hemos señalado, creemos haber cumplido cabalmente con nuestro objetivo principal, que era hacer un reconocimiento orientador y cuantificado del universo de la folletería mexicana del siglo XIX. Asimismo, nos habíamos propuesto desbrozar su contenido, analizar cualitativamente sus posibilidades de utilización. Avanzadas estas dos tareas, aunque no con- 


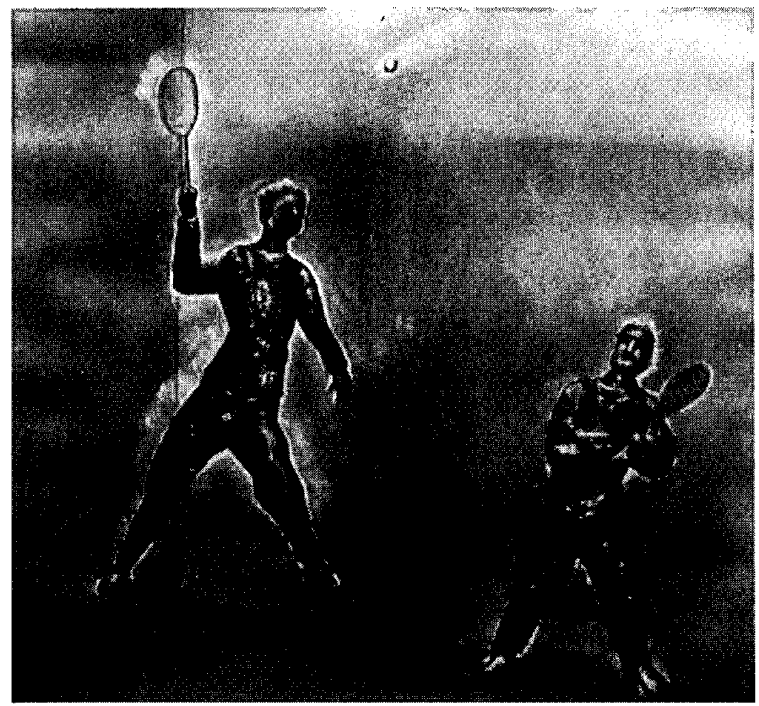

cluidas, llegamos al convencimiento de que los folletos no fueron en el México decimonónico un tipo de publicaciones secundarias y que, considerados como un universo integrado y no como fragmentos inconexos, son una valiosa fuente de información sobre las temáticas que realmente preocuparon a nuestros abuelos y los impulsaron a manifestar su parecer. Vistos así, ofrecerán por un buen tiempo "tela de donde cortar"

\section{BIBLIOGRAFÍA}

-Arenal Fenochio, Jaime del, "Hacia el estudio de la folletería jurídica mexicana, 1851-
1910", Cuadernos del Instituto de Investigaciones Juridicas, año 2 , núm. 4 , enero-abril 1987, Instituto de Investigaciones Jurídicas-UNAM.

-Bachman, John, E., "Los panfletos de la independencia mexicana", Historia Mexicana, vol. xx, núm. 4, abril-junio 1971, pp. $522-538$

-Dublán y Lozano, Manuel, Legislación mexicana, o colección completa de las disposiciones legislaivas expedidas desde la independencia de la república, ordenada por Manuel Dublán y José María Lozano, edición oficial, Imprenta de Comercio de Dublán y Chávez a cargo de M. Lara (hijo), México, 1876-1912.

-Fernández de Lizardi, José Joaquín, Obras, prólogo y notas de Rosa Ma. Palazón Mayoral, UNAM, México, 1995, vol. XIII. 